\title{
Possible effects of dietary $n-6$ series polyunsaturated fatty acids on the development of immune dysfunction and infection
}

\author{
By V. Utermohlen and M. A. M. Tucker, Division of Nutritional Sciences, \\ Cornell University, Ithaca, New York 14853, USA
}

Dietary polyunsaturated fatty acids (PUFA) of the $n-6$ series, in particular linoleic and arachidonic acids, are of great interest to the nutritionist because they are essential for humans and other simple-stomach mammalian species. They serve as the precursor for a host of biologically active compounds, and participate in the maintenance of normal membrane function. Recently, the general population has been encouraged to substitute PUFA for saturated fatty acids in their daily diets, in the hope of reducing the risk of cardiovascular disease. While, for most people consuming a Western-type diet, $n-6$ PUFA may account for 7-15\% of energy intake, persons on total parenteral nutrition may receive as much as $50 \%$ of their energy as $n-6$ PUFA. An as yet unanswered question has been whether consumption of high levels of $n-6$ PUFA may encourage the development of infection, and particular Gram negative infection. Fig. I presents in diagrammatic form the hypotheses on which such a suggestion may be based.

\section{Effect of linoleic acid on the growth of Gram negative bacteria}

In studies involving the use of a fermenting system to simulate gut conditions, Michelson \& Klipstein (1975) found that the addition of linoleic acid encouraged

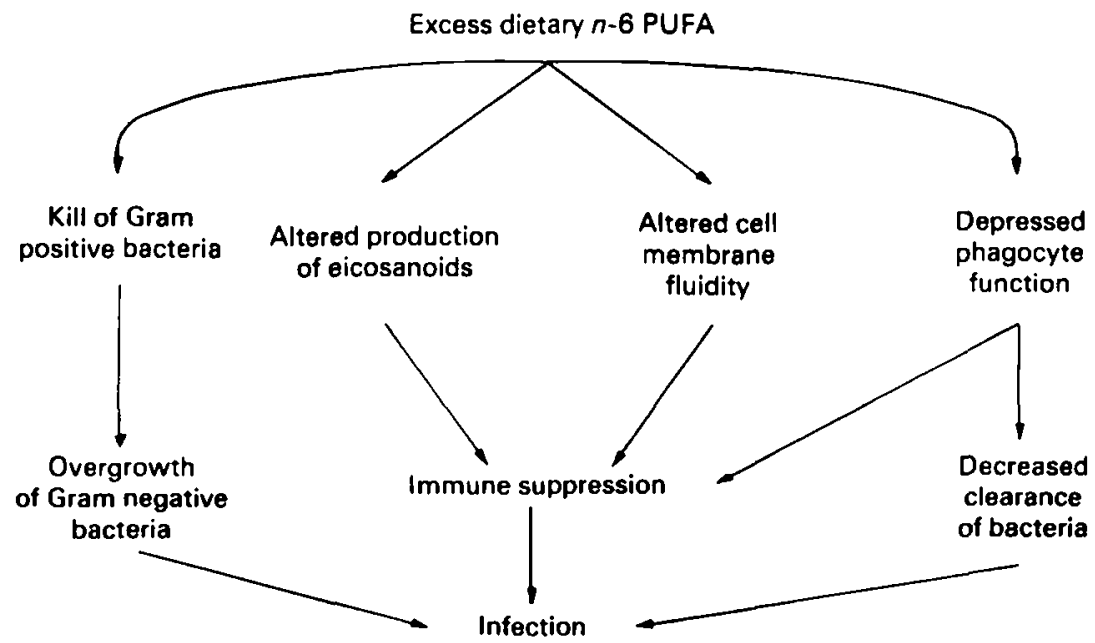

Fig. I. Possible mechanisms whereby $n-6$ polyunsaturated fatty acids (PUFA) may cause increased susceptibility to infection. 
the overgrowth of Klebsiella pneumoniae at the expense of Lactobacillus acidophilus. The rationales for performing this experiment included the observations that: (a) Gram negative bacteria are prominent in the small intestine in tropical sprue, (b) tropical sprue in Puerto Rico often follows the consumption of large amounts of pork which is rich in $n-6$ PUFA (Klipstein \& Corcino, 1974). These authors therefore have suggested that dietary PUFA promote the growth of Gram negative bacteria and thus contribute to the pathogenesis of the disease. PUFA appear to be bacteriocidal through their ability to disrupt cell walls and membranes via a detergent-like effect. Kodicek ( 1949 ) noted that unsaturated fatty acids inhibited the growth of Gram positive bacteria and similar results were obtained by Kabara et al. (1972). Sheir \& Freese (1973) have suggested that the lipopolysaccharide layer around Gram negative bacteria protects them against disruption by long-chain fatty acids. Thus the predominance of Gram negative bacteria when long-chain fatty acids are present may be due to elimination of competing Gram positive bacteria from a mixed flora. Under normal circumstances most tissues exposed to bacteria (skin, gut, lungs) can maintain a balanced flora. However, when tissues are flooded with $n$ - 6 PUFA as may be the case of the gut in tropical sprue, Gram negative bacteria can preponderate, activate complement and thus macrophages via their lipopolysaccharide coat (Gewurz et al. I968), and initiate the process of inflammation and infection Parker (1984).

\section{Effects of n-6 PUFA on immune function}

A second means whereby $n-6$ PUFA may promote the development of infection is through changes in immune function. The mechanism appears to depend on the route of PUFA administration. When PUFA are delivered parenterally in the form of a vegetable-oil emulsion, immune suppression appears to be due primarily to macrophage dysfunction. The oil microdroplets apparently are taken up by phagocytic cells, which become 'clogged' and are no longer capable of phagocytosis (Passwell et al. 1976; Fischer et al. 1979). In experimental animals this paralysis of the reticuloendothelial system has been shown to lead to the development of sepsis (Fischer et al. 1980; Hamawy et al. 1985). Evidence that in humans sepsis is more frequent or more severe in patients receiving lipid emulsions as part of a parenteral nutrition regimen is lacking (Allen, I 978 ; Mullin \& Kirkpatrick, I 983 ).

When PUFA are delivered as food via the oral route, they appear in the plasma and in membranes of circulating lymphoid cells quite rapidly. Feeding of an extra 1 7-2 $\mathrm{g}$ linoleic acid daily in the form of sunflower or safflower oil results in significant increases in the proportion of linoleic acid in both plasma and lymphoid cells (Tsang et al. 1976; Boyar, 1979). The presence of relatively large amounts of these fatty acids can be expected to alter immunocyte function in at least two ways: via an increase in membrane 'fluidity' and via the production of immunologically-active eicosanoids.

PUFA exist in immunocyte (lymphocyte, macrophage) membranes both as free fatty acids and as acyl chains of membrane phospholipids. In these positions, they appear to confer 'fluidity' to the membrane, and render it more 'sol-like' than 
'gel-like' (Karnovsky et al. 1982). Much of the work done to link immune responsiveness to changes in membrane fluidity has involved modulation of the ratio, cholesterol:phospholipid in the membrane (for review, see Traill \& Wick, 1984). Blastogenesis studies have suggested that there is a biphasic response, with one effect at low doses of exogenous cholesterol or phospholipid and the opposite effect at high doses. Whether these effects can be attributed to changes in membrane fluidity, in membrane characteristics or in the availability of cholesterol is debatable. At the same time, target-cell immune cytolysis by complement plus antibody appears to be directly dependent on membrane fluidity. By contrast, T-cell-mediated cytolysis is enhanced when the membrane of the target cell is highly negatively charged through the presence of polar lipids in the outer leaflet of the membrane.

Parallel, although less extensive, results have been obtained with linoleate and arachidonate. Karnovsky et al. (1982) classify linoleate and arachidonate, with oleate, as class A fatty acids, which partition preferentially into the sol phase of the membrane. The addition of these fatty acids to lymphocytes increases the area of the membrane in the sol state and causes cytoskeletal reorganization. The latter changes the membrane distribution of cell surface proteins and the ability of the proteins to move in the plane of the membrane (Karnovsky et al. 1982; Utermohlen et al. 1986). Vertical mobility of proteins and their presentation on the cell surface may be affected by membrane incorporation of $n-6$ PUFA, although the effect may be modulated by differences in the degree of incorporation between the inner and outer membrane (Utermohlen et al. 1986). Unsaturated fatty acids may alter cell transport functions, which in turn may alter the rate of nutrient entry into the cell, and thus regulate cell division (Burns et al. 1979; Poon et al. 1981; Utermohlen et al. 1986).

Changes in any or all of these functions may be expected to alter immune responsiveness, although again a direct link between these changes and actual measures of immune responsiveness has been difficult to establish. Nevertheless the notion that, for example, changes in membrane fluidity may alter the presentation of surface antigens of importance in triggering immune responses or in subserving cell-cell or cell-lymphokine interaction, as proposed by Shinitzky \& Sourojon (1979), is an attractive one.

The roles of the eicosanoids (prostaglandins, leukotrienes and other peroxy metabolites of $n-6$ PUFA) in modulating immune function are the subject of considerable research and debate (for review, see Gurr, I983; Johnston \& Marshall, 1984). It appears that these metabolites may have differing effects depending on the cellular target and the dose. As yet we know little about the mechanism of action of the eicosanoids and about the regulation of their production.

An example of the complexity of the effects of eicosanoids is the role of $\mathrm{LTB}_{4}$, which can activate suppression of lymphocyte blastogenesis (Rola-Pleszczynski et al. 1982) while it enhances natural cytotoxicity (Rola-Pleszczynski et al. 1983) and the production of pro-inflammatory IL-I (Rola-Pleszczynski \& Lemaire, I986). $\mathrm{PGE}_{2}$ also causes suppression of cell-mediated responses such as 
blastogenesis due to IL-I (Kunkel et al. 1985 ). It should be noted, however, that these generalizations may be applicable only for certain dose ranges and their validity may depend on the initial state of the cells before the administration of the eicosanoid. We have found, for example, that $\mathrm{PGE}_{2}$ may enhance suppressed migration responses to paramyxoviral antigens, while it suppresses enhanced function (Utermohlen et al. 1980). From these findings it would be difficult to determine whether or not dietary $n-6$ PUFA can be expected to lead to increased infection through immune suppression.

\section{The relation of n-6 PUFA-induced changes in immune function to infection}

To date there have been no studies showing that the consumption of a high- $\boldsymbol{n}-\boldsymbol{6}$ PUFA diet will lead to the development of opportunistic infections, despite the theoretical grounds for such a development. We reasoned, therefore, that an initial immunologic insult may be required before infection can develop in animals consuming high doses of PUFA.

One of the most effective insults in causing immune suppression is thermal injury. Furthermore, the burn site is prone to the development of Gram negative infection. A diet high in linoleic acid can diminish both cell-mediated immune responses and the opsonic index in guinea-pigs which have undergone $30 \%$ total body surface area burn (Trocki et al. 1986).

In a study of rats undergoing a $15 \%$ body surface area scald, we found that animals receiving a $155 \mathrm{~g}$ maize oil/ $\mathrm{kg}$ diet developed a more rapid and prolonged immunosuppression, as measured by splenocyte mitogen responsiveness, than animals consuming a $44.5 \mathrm{~g}$ maize oil/ $/ \mathrm{kg}$ diet. At 3 and 4 weeks post burn, four of six animals on the high-maize-oil diet, but only two of five animals on the low-maize-oil diet grew Gram negative bacteria (primarily Pseudomonas sp.) from their wounds. These differences occurred despite the fact that there was no obvious difference in nutritional status (weight, food consumption) between the two groups of animals (M. A. M. Tucker and V. Utermohlen, unpublished results).

The slightly increased frequency of Gram negative infections in animals receiving the high-maize-oil diet may be due to changes in immune function. However, the possibility that the wound exudate contained higher amounts of linoleic acid, which would promote overgrowth of Gram negative bacteria, remains.

\section{Conclusion}

The experiments which link n-6 PUFA to susceptibility to infection are few, but highly suggestive. There is a strong theoretical basis for anticipating such a link. It therefore behoves the person who is administering $n-6$ PUFA at high doses (e.g. to patients receiving total parenteral nutrition with lipids as a vegetable-oil emulsion) to be alert for an increased possibility of infection. Clinical observations, coupled with ongoing exploration of the mechanisms of PUFA-induced enhancement of growth of Gram negative bacteria, should provide a means of judging whether a high n-6 PUFA intake would be detrimental in conditions which might lead to sepsis. 


\section{REFERENCES}

Allen, J. R. (1978). In Advances in Parenteral Nutrition, pp. 339-377 [I. D. A. Johnston, editor]. Baltimore, MD: University Park Press.

Boyar, A. P. (1979). A study of the serum fatty acids, dietary fats, and cell-mediated immune responses in non-hospitalized patients with multiple sclerosis before and during oil supplementation. PhD Thesis, Cornell University, Ithaca.

Burns, C. P., Lutteneger, D. G. \& Dudley, D. T. (1979). Cancer Research 39, 1726-1 730.

Fischer, G. W., Hunter, K. W. \& Wilson, S. R. (1979). Pediatric Research 13, 494-497.

Fischer, G. W., Wilson, S. R., Hunter, K. W. \& Mease, H. D. (1980). Lancet ii, 819-820.

Gewurz, H., Shin, H. S. \& Mergenhagen, S. E. (1968). Fournal of Experimental Medicine 128, 1049-1057.

Gurr, M. I. (1983). Progress in Lipid Research 22, 257-289.

Hamawy, K. H., Moldawer, L. L. \& Georgieff, M. (1985). Fournal of Parenteral and Enteral Nutrition 9, 559-565.

Johnston, D. V. \& Marshall, L. A. (1984). Progress in Food and Nutrition Science 8, 3-25.

Kabara, J. J., Swieczowski, D. M., Conley, A. J. \& Truant, J. P. (1972). Antimicrobial Agents in Chemotherapy 2, 23-28.

Karnovsky, M. J., Kleinfeld, A. M. \& Hoover, R. L. (1982). Annals of the New York Academy of Science 401, 61-75.

Klipstein, F. A. \& Corcino, J. J. (1974). American Journal of Tropical Medicine and Hygiene 23, $1189-1196$.

Kodicek, E. (1949). Symposia of the Society of Experimental Biology 3, 217.

Kunkel, S. L., Chesue, S. W., Spengler, M. \& Gerr, J. (1985). In The Physiologic, Metabolic and Immunologic Actions of Interleukin-1. Progress in Leukocyte Biology, vol. 2, pp. 297-307 [M. J. Kluger, J. J. Oppenheim and M. C. Powanda, editors]. New York: A. R. Liss.

Michelson, M. J. \& Klipstein, F. A. (1975). Infection and Immunology 12, 1 12 I-1 126.

Mullin, T. J. \& Kirkpatrick, J. R. (1983). Archives of Surgery $118,176-180$.

Parker, C. W. (1984). In Fundamental Immunology, pp. 721-722 [W. E. Paul, editor]. New York: Raven Press.

Passwell, J. H., David, R. \& Katznelson, D. (1976). Archives of Disease in Childhood 51, 366-368.

Poon, R., Richard, J. M. \& Clark, W. R. (1981). Biochimica et Biophysica Acta 649, 58-66.

Rola-Pleszczynski, M., Borgeat, P. \& Sirois, P. (1982). Biochemical and Biophysical Research Communications 108, I $531-1537$.

Rola-Pleszczynski, M., Gagnon, L. \& Sirois, P. (1983). Biochemical and Biophysical Research Communications $113,531-537$.

Rola-Pleszczynski, M. \& Lemaire, I. (1986). Fournal of Immunology (In the Press).

Sheir, C. W. \& Freese, E. (1973). Fournal of Bacteriology $115,869-875$.

Shinitzky, M. \& Sourojon, M. (1979). Proceedings of the National Academy of Sciences, USA 76, $443^{8}-444^{\circ}$.

Traill, K. N. \& Wick, G. (1984). Immunology Today 5, 70-76.

Trocki, O., Saito, H. \& Gonce, S. J. (1986). Fournal of Parenteral and Enteral Nutrition 10, Suppl. 55 .

Tsang, W. N., Belin, J. \& Monro, J. (1976). Fournal of Neurology, Neurosurgery and Psychiatry 39, $767-771$.

Utermohlen, V., Besner, G. \& Ginsparg Berkowitz, M. (1980). Clinical Immunology and Immunopathology 16, 324-335.

Utermohlen, V., Kilburn, S., Kiselis, I. \& Mao, D. (1986). In Proceedings of XIII International Congress of Nutrition, Brighton [T. G. Taylor, editor]. London: John Libbey (In the Press). 